\title{
Over-the-scope clip (OTSC)-induced colonic obstruction necessitating sigmoid resection
}

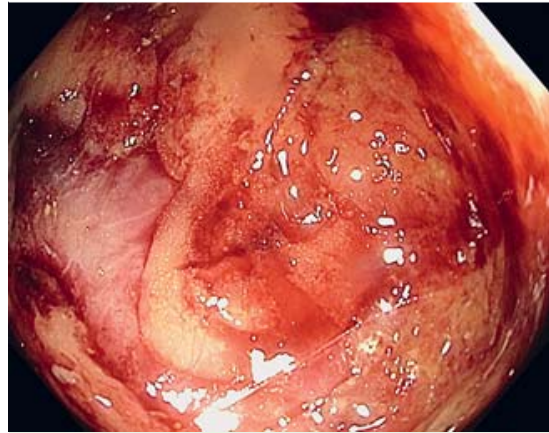

Fig. 1 Endoscopic view showing mesenteric fat within the abdominal cavity through a perforation of the sigmoid colon.

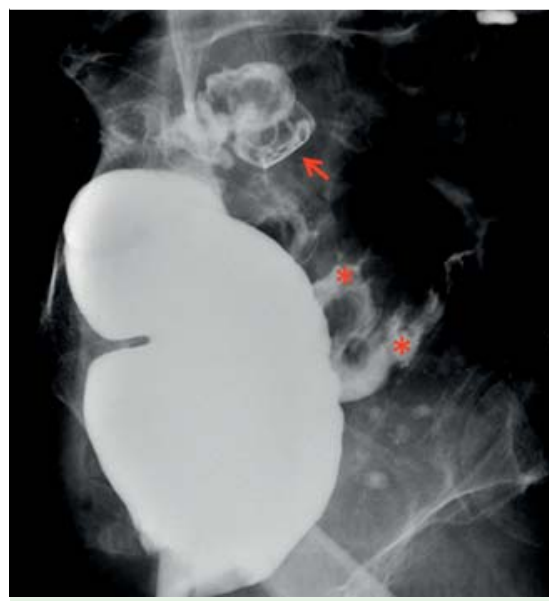

Fig. 3 Contrast enema 2 days after application of the over-the-scope clip (OTSC) showing the narrowed clip site (arrow) and only minimal flow of the contrast agent beyond this point (asterisks).

A 75-year-old patient was admitted to our hospital for investigation of chronic anemia with a hemoglobin value of $6.6 \mathrm{~g} / \mathrm{dL}$. The patient's medical history included a mitral valve replacement and a kidney transplant 10 years and 8 years ago, respectively. A diagnostic endoscopy was performed and sigmoid diverticulosis with signs of postinflammatory bowelwall sclerosis was observed as the colonoscope was being advanced to the cecum. As the endoscope was being withdrawn, a large perforation was seen in the sigmoid colon ( $\bullet$ Fig. 1). As the perforation had not been visible during introduction of the colonoscope, it seems most likely
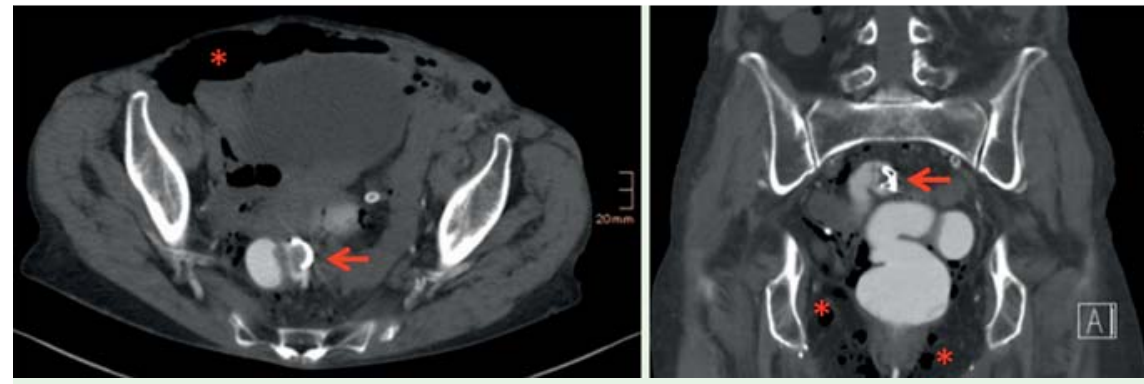

Fig. 2 Computed tomography (CT) scan 1 hour after the over-the-scope clip (OTSC) had been applied showing that the contrast agent stops at the site of the OTSC (arrow) and there is free abdominal air (asterisks).

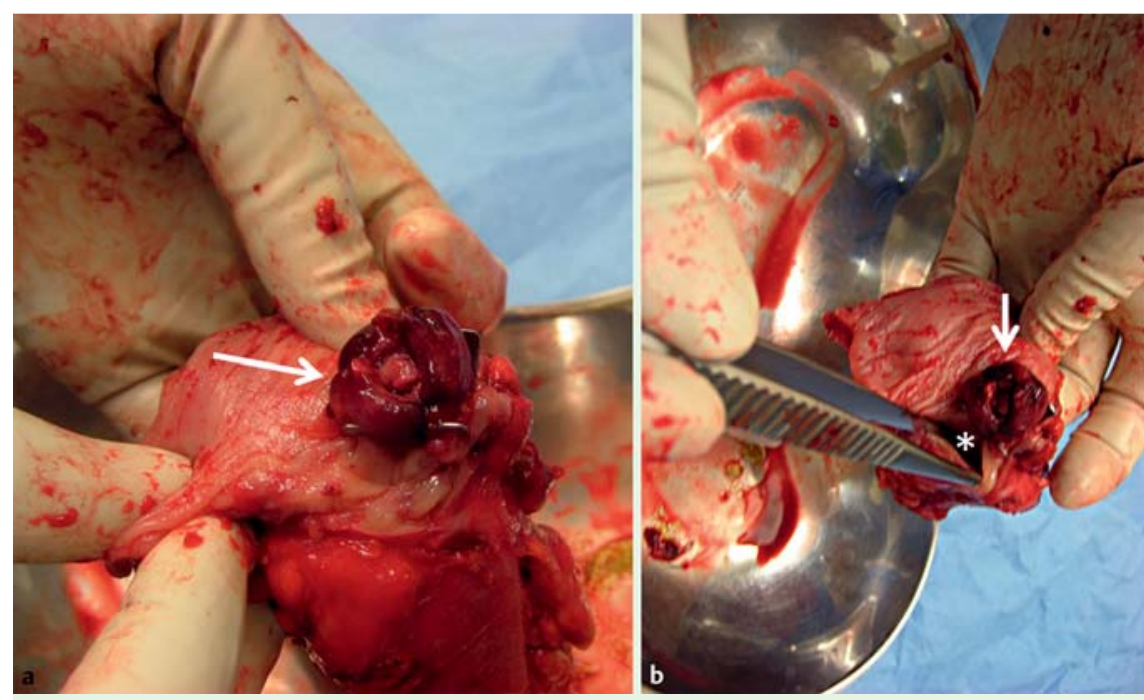

Fig.4 Gross appearance of the opened sigmoid resection specimen showing: a an endoluminal view with a prominent tissue bulge caused by the over-the-scope clip (OTSC; arrow) clearly visible; $\mathbf{b}$ a forceps placed within the residual colonic lumen (asterisk), which has a diameter comparable to that of the tissue bulge (arrow).

that it occurred during advancement of the scope to the cecum.

A 14/6t over-the-scope clip (OTSC; Ovesco Endoscopy, Tübingen, Germany) was mounted on the colonoscope, which was advanced to the perforation site. The two perforation margins were easily grasped with the Twin Grasper (Ovesco Endoscopy) and were pulled into the transparent cap. Additional suction was then applied to the tissue that had been grasped within the cap before the clip was released.

An endoscopic check showed satisfactory closure of the perforation but that the colonic lumen was considerably narrowed. Even using a gastroscope $(10 \mathrm{~mm}$ in diam- eter), it was impossible to pass the scope beyond the narrowed section at the site of the clip. Computed tomography (CT) scanning with a contrast enema did not reveal a residual leak, but in keeping with the endoscopic appearance, there was total blockage of the flow of contrast agent at the level of the OTSC ( $\bullet$ Fig.2). A contrast enema 2 days later showed a similar situation with clip-induced subtotal stenosis ( $\bullet$ Fig. 3 ).

Because of the clinical signs of bowel obstruction (bowel distension, high-volume secretion via the gastric tube, and pain), the patient underwent open sigmoid resection 2 days after application of the clip. 
On the resection specimen, the clip was causing a visible tissue bulge, which readily explained the mechanical bowel obstruction ( $\bullet$ Fig. 4$)$.

OTSCs are increasingly used in the treatment of acute gastrointestinal perforations and fistulas $[1,2]$. To the best of our knowledge, this is the first report describing a mechanical clip-induced bowel obstruction. If the perforation margins can be grasped with the Twin Grasper and satisfactorily pulled into the cap, it may not always be advisable to mobilize as much tissue as possible into the cap. Care needs to be taken applying additional suction, especially when there is underlying postinflammatory bowel-wall sclerosis or a small gastrointestinal lumen.

Endoscopy_UCTN_Code_CPL_1AJ_2AI

Competing interests: None

\section{Andreas Fischer, Hans-Jürgen Richter-Schrag}

Interdisciplinary Endoscopy, Department of General and Visceral Surgery, Department of Medicine II, University Hospital Freiburg, Freiburg, Germany,

\section{References}

1 Haito-Chavez Y, Law JK, Kratt T et al. International multicenter experience with an overthe-scope clipping device for endoscopic management of GI defects (with video). Gastrointest Endosc 2014; 80: 610-622

2 Mennigen R, Senninger N, Laukoetter MG Novel treatment options for perforations of the upper gastrointestinal tract: endoscopic vacuum therapy and over-the-scope clips. World J Gastroenterol 2014; 20: 7767 -7776

\section{Bibliography}

Dol http://dx.doi.org/

10.1055/s-0034-1391425

Endoscopy 2015; 47: E157-E158

(c) Georg Thieme Verlag KG

Stuttgart · New York

ISSN 0013-726X

\section{Corresponding author}

\section{Andreas Fischer, MD}

University Hospital Freiburg Interdisciplinary Endoscopy

Department of General and Visceral Surgery, Department of Medecin II

Sir Hans A. Krebs Str.

\section{Freiburg}

\section{Germany}

Fax: +49-761-27025411

andreas.fischer@uniklinik-freiburg.de 